\title{
Simulation of spiculated breast lesions
}

\author{
Premkumar Elangovan*a , Faisal Alrehily ${ }^{\mathrm{b}}$, R Ferrari Pinto ${ }^{\mathrm{a}}$, Alaleh Rashidnasab ${ }^{\mathrm{a}, \mathrm{c}}$, David R \\ Dance $^{\mathrm{b}, \mathrm{d}}$, Kenneth C Young ${ }^{\mathrm{b}, \mathrm{d}}$, Kevin Wells ${ }^{\mathrm{a}}$ \\ ${ }^{a}$ Centre for Vision, Speech and Signal Processing, University of Surrey, Guildford, GU2 7XH, UK \\ ${ }^{\mathrm{b}}$ Department of Physics, University of Surrey, Guildford, GU2 7XH, UK \\ ${ }^{\mathrm{C}}$ Institute of Nuclear Medicine, University College London, NW1 2BU, UK \\ ${ }^{\mathrm{d}}$ NCCPM, Royal Surrey County Hospital, Guildford, Surrey, GU2 7XX, UK
}

\begin{abstract}
Virtual clinical trials are a promising new approach increasingly used for the evaluation and comparison of breast imaging modalities. A key component in such an assessment paradigm is the use of simulated pathology, in particular, simulation of lesions. Breast mass lesions can be generally classified into two categories based on their appearance; nonspiculated masses and spiculated masses. In our previous work, we have successfully simulated non-spiculated masses using a fractal growth process known as diffusion limited aggregation. In this new work, we have extended the DLA model to simulate spiculated lesions by using features extracted from patient DBT images containing spiculated lesions. The features extracted included spicule length, width, curvature and distribution. This information was used to simulate realistic looking spicules which were attached to the surface of a DLA mass to produce a spiculated mass. A batch of simulated spiculated masses was inserted into normal patient images and presented to an experienced radiologist for review. The study yielded promising results with the radiologist rating $60 \%$ of simulated lesions in 2D and 50\% of simulated lesions in DBT as realistic.
\end{abstract}

Keywords: virtual clinical trials, simulated lesions, simulated masses, DLA, modelling tools, breast cancer.

\section{INTRODUCTION}

National breast cancer screening programmes have been introduced in many countries for early detection and treatment of the breast cancer ${ }^{1}$. Although 2D-mammography has been the main imaging methodology in screening, new approaches such as digital breast tomosynthesis (DBT) have been proposed. However, the optimal use of such technology remains an open question.

Clinical trials including human subjects are the conventional approach to answering questions of utility and optimality. This approach is cumbersome and time-consuming and cannot match the rapid pace of technological development. Computerized modelling tools ${ }^{2}$ can be used to complement or replace conventional clinical trials and such use is generally referred to as a 'virtual clinical trial'. Virtual clinical trials involve simulation of a large number of images to conduct quantitative evaluation and comparison studies of different imaging modalities and system designs. However, virtual clinical trials rely on the availability of a range of realistic looking pathologies to produce images that have comparable appearance to those of patient images produced in an equivalent real screening setting.

Cancer anomalies primarily manifest in a women's breast in the form of masses and micro-calcification clusters. Masses can be broadly classified into two types based on their appearance: (1) non-spiculated masses and (2) spiculated masses. Figure 1 shows examples of spiculated and non-spiculated lesions. In our previous work ${ }^{3,4}$, we simulated and validated non-spiculated masses using the method known as DLA (Diffusion Limited Aggregation). This is a fractal-like growth process to generate 3D irregular lesion-like structures. This complements prior work ${ }^{3}$ using random walk that could generate benign masses and cystic-like structures. In this work, we use a biologically inspired approach to extend the solid DLA mass model to produce simulated spiculated lesions. In contrast to prior work using geometrical spicule simulation $^{5,6}$, our approach involves extraction of various features from pathology confirmed spiculated cancers and using these as guidelines to characterize and append spicules onto the surface of solid DLA masses.

*p.elangovan@surrey.ac.uk; 


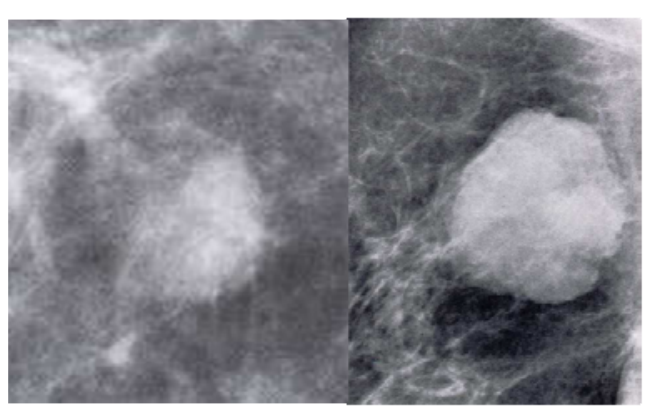

(a)

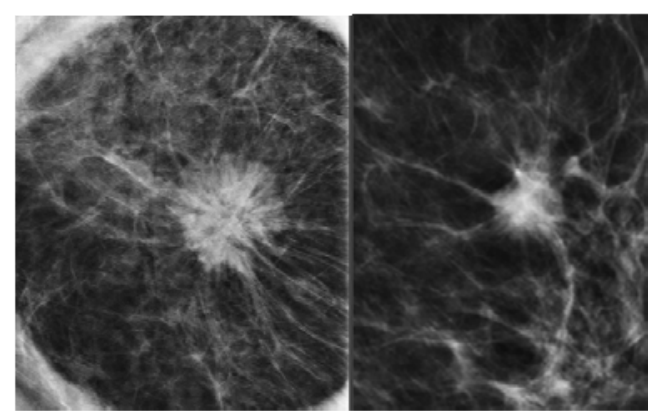

(b)

Figure 1. (a) Examples of real non-spiculated and (b)spiculated masses.

\section{MATERIALS AND METHODS}

This section starts with a brief explanation of DLA solid mass generation technique. Following this, the method for simulating spicules onto the surface of the solid mass is explained.

\subsection{DLA solid mass simulation}

Solid masses generally contain a distinct core with regular and/or irregular boundaries, for which the DLA method can be used. The simulation process starts with constructing a bounding box to hold the lesion structure and placing a particle in the center of the box. Then, particles are launched from edges of the bounding box. These particles random walk towards the center and gradually agglomerate when it comes in contact with previous particles within the bounding box to form a 3D lesion like structure. This process is repeated until the required size/density is achieved or until a predefined terminal condition is reached. One of the fundamental advantages of the DLA method is that there are many parameters that can be controlled to simulate particular characteristics (size, texture and density) of the masses. The DLA parameters include: number of launching points, step size of random walk and sticking probability.

\subsection{Simulation of spicules}

Spiculated lesions can be generally classified into four categories depending on the appearance and distribution of the spicules: (1) medium and thick branches; (2) stellate; (3) hairy uniformly distributed branches; and (4) grouped branches. The spiculated masses were simulated by first wrapping a 3D triangular mesh around a solid DLA lesion and then appending spicule-like structures normal to the mesh surface. In order to simulate spicules with realistic curvature and distribution, various features were extracted from patient DBT images and used as a guideline for generating spicules. This was performed by manually scrolling through DBT planes and marking the points at which spicules appear infocus. Then, a 3D spline interpolation was used to connect the selected points to produce a 3D skeleton of each spicule. The resulting 3D "skeleton" was then converted into thin horns by a series of dilation operations and placed on the surface of a DLA mass. Finally, the 3D geometric horns were filled up with fine fiber like structures in order to provide the correct density and internal texture. This fibrotic characteristic can be observed in specimen X-ray images of real spiculated lesions. Parameters such as thickness, length, porosity, diameter and eccentricity of the resulting conical structures were adjusted until a realistic looking spiculated mass was produced. The spiculated lesion generation process is illustrated in Figure 2.

Various measurements were performed on the spiculated lesions seen in clinical images including (1) length of spicules and (2) apparent width of spicules at different locations along the spicule length. This information was used to drive the parameter selection for simulating 3D spiculations with realistic curvature. Figure 3 shows the measured spicule thickness plotted as a function of spicule distance from the base. 

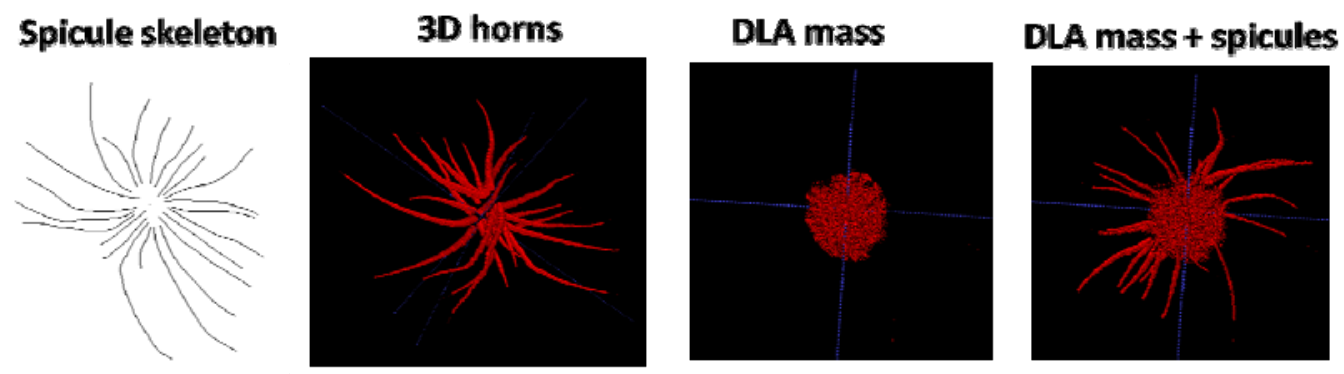

(a)
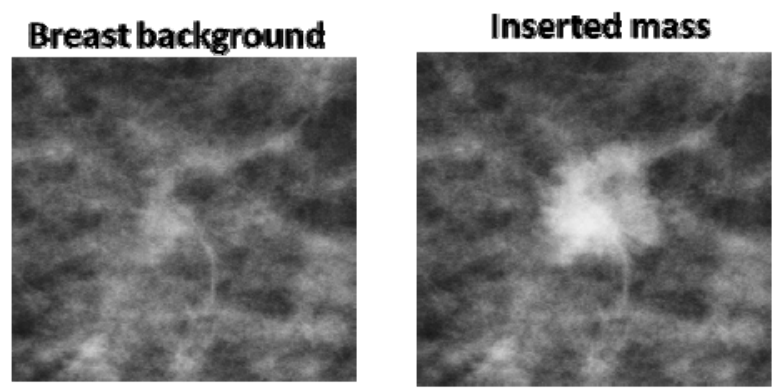

Spicules attached

(b)

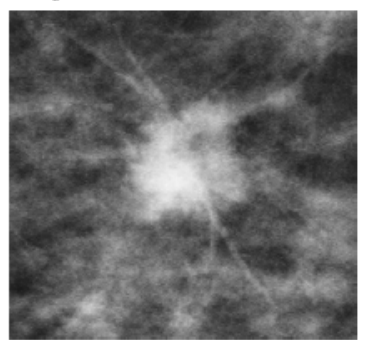

Figure 2. (a) Spiculated lesion generation process and (b) insertion into 2D-mammograms.

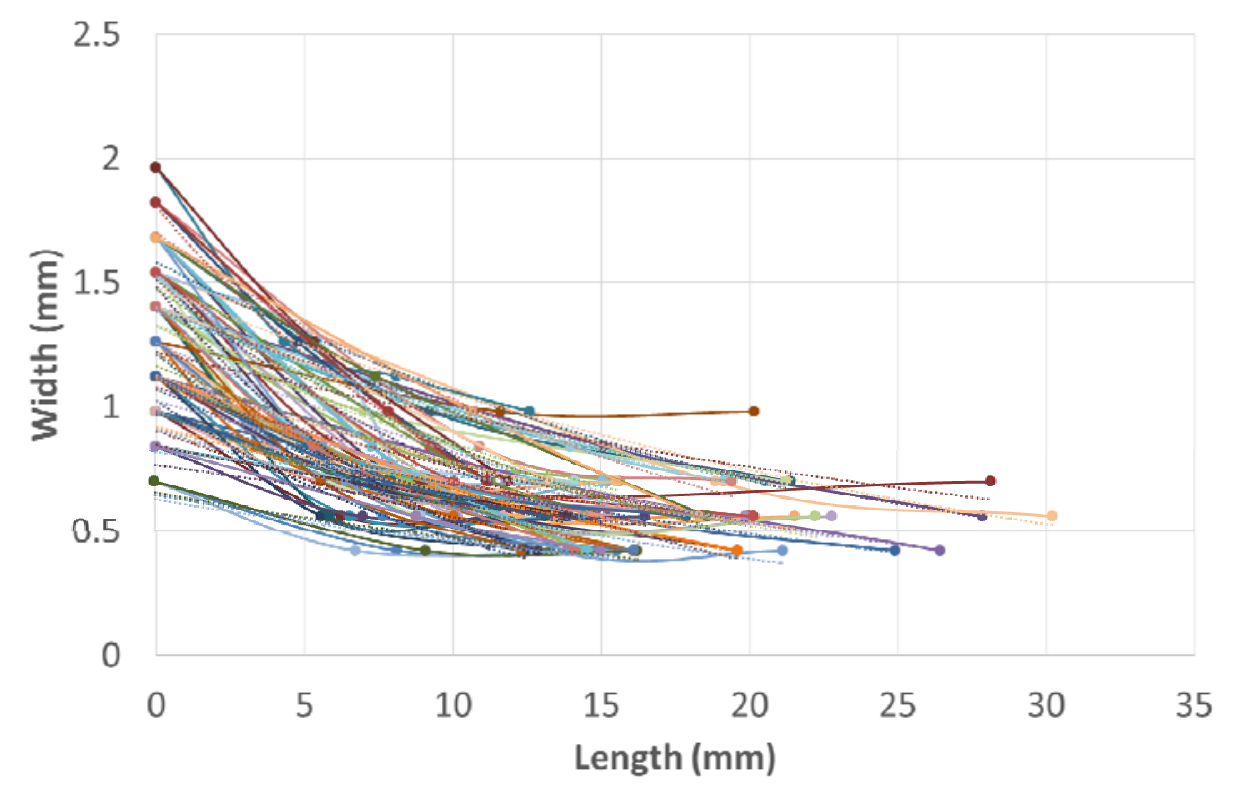

Figure 3. Plot illustrating measured spicule thickness vs. distance from the core. 


\subsection{D mass insertion into real images}

In order to evaluate the appearance of the spiculated masses, the masses were inserted into normal patient images obtained on a Hologic Selenia Dimensions 3D system (Hologic Inc, Bedford, Massachusetts, USA) using a physicsbased insertion technique ${ }^{2}$. A brief summary of this technique is given below. The Hologic system while operating in 'combo' mode produces a 2D image with a pixel pitch of 70um and 15 tomosynthesis projections with a pixel pitch of 140um acquired over the angular range $+7.5^{\circ}$ to $-7.5^{\circ}$. First, the system acquisition parameters are estimated from the DICOM header of the clinical image into which the lesion is to be inserted. Then, the $3 \mathrm{D}$ volume containing the simulated lesion and the insertion location are fed into a ray tracing model along with the estimated system acquisition parameters. The ray tracing tool produces two sets of images one with and another without the lesion for an assumed breast thickness and glandularity. The composition of the mass was assumed to be equivalent to that of glandular tissue and attenuation coefficients were calculated based on the elemental composition information reported in Hammerstein et $a l^{7}$. Then the images are blurred using the experimentally measured $\mathrm{MTF}^{8}$ of the system. Then, an attenuation template is created from the images which correspond to the relative increase in the attenuation due to the inclusion of the lesion at the insertion site. The template is multiplied with the normal image after removal of scatter (estimated from Monte Carlo simulations ${ }^{9}$ ) and any other known offsets to simulate the appearance of the lesion in the image. After modification of the primary image, the scatter and the known pixel offsets are reinstated. This process is repeated for the 2D projection and 15 tomosynthesis projections. Finally, the unprocessed 2D image is reconstructed using a manufacturer-specific image-processing tool and the tomosynthesis projections are reconstructed into DBT planes using the Hologic reconstruction software based on a filtered back projection algorithm. Further details of the method are given in Elangovan $e t l^{2}$.

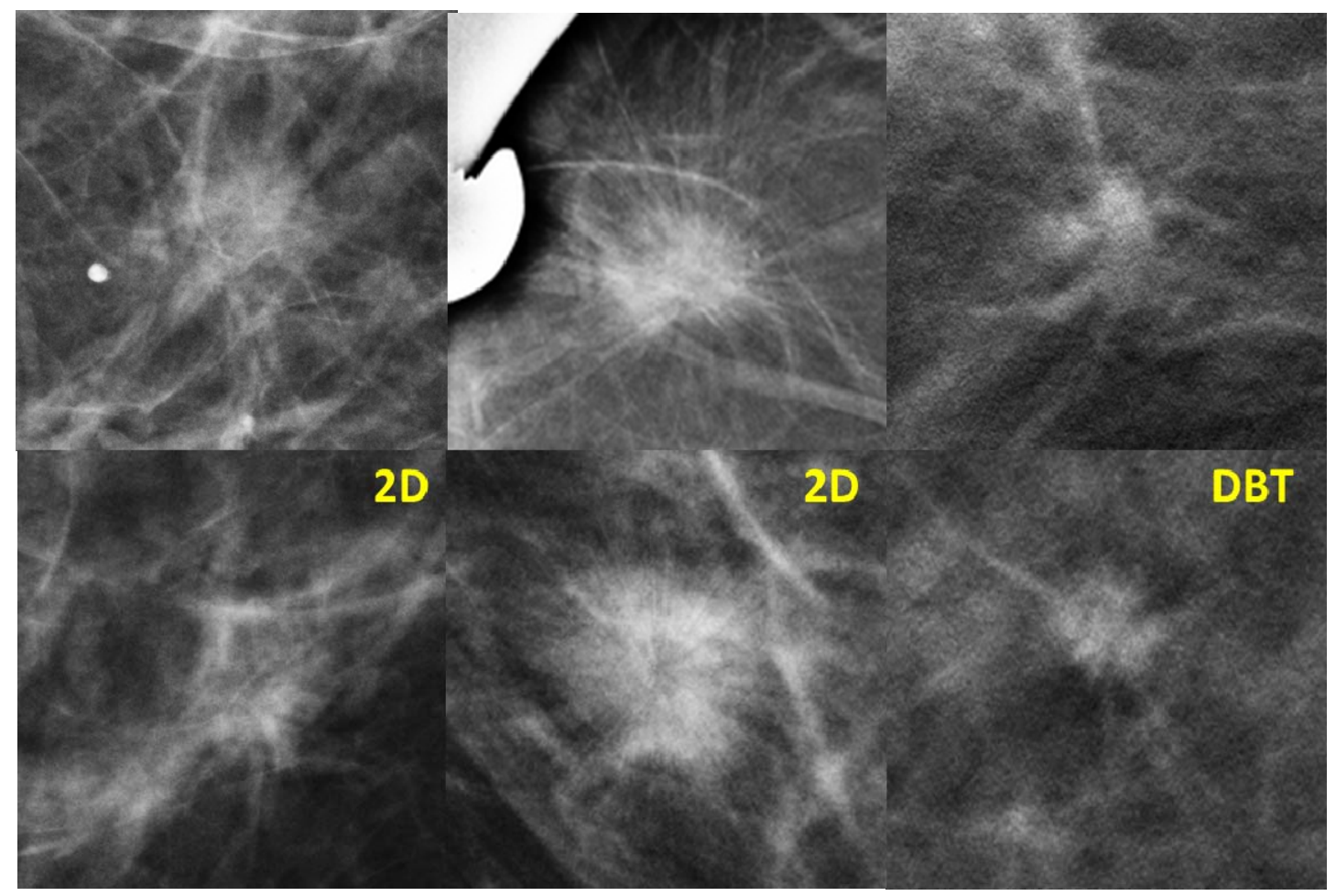

Figure 4. Examples of real (top row) and simulated (bottom row) spiculated masses. 


\section{RESULTS \& DISCUSSION}

A batch of simulated spiculated lesions was inserted into normal patient images for a pilot study involving a single experienced breast radiologist ( $>10$ years). A total of 13 masses were simulated and inserted into 2D and DBT images. The size of the simulated masses ranged between $7-9 \mathrm{~mm}$ and the breast region displayed was limited to an area of $3 \times 3 \mathrm{~cm}^{2}$ with the lesion located in the center. The images were interleaved with real spiculated lesions resulting in a total of 26 images for each modality (2D and DBT). The radiologist was asked to make a preliminary assessment of images using a six point scale, 1: Extremely confident that the mass is real, 2: Moderately confident that the mass is real, 3: Slightly confident that the mass is real, 4: Slightly confident that the mass is simulated, 5: Moderately confident that the mass is simulated, 6: Extremely confident that the mass is simulated. Figure 4 shows some simulated lesions used in the study alongside real lesions. The radiologist rated $60 \%$ of simulated lesions in 2D and 50\% of simulated lesions in DBT as probably or possibly real (scales 3-4). The radiologist was able to correctly identify all the 2D and DBT real images in the dataset.

\section{CONCLUSION}

In this paper, we have detailed a method for simulating lesions with spicule like branches. The simulated spicules were created taking account of the features of real spiculated lesions in patient DBT images. The results of the pilot validation study indicate that the method produces some realistic looking spiculated lesions. The method is fast and efficient. Once a database of spiculated parameters has been constructed and a set of DLA cores are available, spiculated masses can be generated in less than a minute and inserted into clinical images making this approach suitable for virtual clinical trials where large numbers of images containing pathology are required. Those images rated as simulated by the observer were found to have an absence of apparent distortion in the core due to spicule growth. The future work will be aimed at incorporating techniques to induce tissue distortion in the simulated lesions.

\section{ACKNOWLEDGEMENTS}

This work is part of the OPTIMAM2 project and is funded by Cancer Research UK. We are grateful for Hologic's assistance with the reconstruction tool. The authors thank colleagues at NCCPM for their invaluable assistance and Dr Vicky Cooke at the Jarvis Breast Screening Centre, Guildford for participating in the observer study and providing feedback.

\section{REFERENCES}

[1] Helvie, M. A., Chang, J. T., Hendrick, R. E. and Banerjee, M., "Reduction in late-stage breast cancer incidence in the Mammography era," Cancer, 1259-2656 (2014).

[2] Elangovan, P., Mackenzie, A., Diaz, O., Rashidnasab, A., Dance, D. R., Young, K. C., Warren, L. M., Shaheen, E., Bosmans, H., Bakic, P. R. and Wells, K., "Development and validation of a modelling framework for simulating 2Dmammography and breast tomosynthesis,” Phys. Med. Biol. 59(15), 4275-93 (2014).

[3] Rashidnasab, A., Elangovan, P., Diaz, O., Mackenzie, A., Young, K. C., Dance, D. R., and Wells, K., "Simulation of 3D DLA masses in digital breast tomosynthesis,” Proc. SPIE 8668, 86680Y (2013).

[4] Rashidnasab, A., Elangovan, P., Yip, M., Young, K. C., Dance, D. R., and Wells, K., "Simulation and assessment of realistic breast lesions using fractal growth models," Phys. Med. Biol. 15, 5613-5626 (2013).

[5] Shaheen, E., De Keyzer, F., Bosmans, H., Dance, D.R., Young, K.C., Van Ongeval, C., "The simulation of 3D mass models in 2D digital mammography and breast tomosynthesis,” Med. Phys. 41(8), 081913-1-17 (2014).

[6] De Sisternes, L., Brankov, J. G., Zysk, A. M., Schmidt, R. A., Nishikawa, R. M., and Wernick, M. N.,"A Computational model to generate simulated three-dimensional breast masses," Med. Phys. 42(8), 1098-1118 (2015).

[7] Hammerstein, G. R., Miller, D, W., White, D. R., Masterson, M. E., Woodard, H. Q. and Laughlin, J. S., "Absorbed radiation dose in mammography," Radiology 130, 485-91 (1979).

[8] Mackenzie, A., Dance, D. R., Workman, A., Yip, M., Wells, K. and Young, K. C., "Development and validation of a method for converting images to appear with noise and sharpness characteristics of a different detector and X-ray system," Med. Phys. 39, 2721-2734 (2012). 
[9] Diaz, O., Dance, D. R., Young, K. C., Elangovan, P., Bakic, P. R. and Wells, K., "Estimation of scattered radiation in digital breast tomosynthesis,” Phys. Med. Biol. 59(15), 4375-90 (2014). 